\begin{tabular}{c} 
Brazilian Journal \\
of Chemical \\
Engineering \\
\hline
\end{tabular}

ISSN 0104-6632

Printed in Brazil

www.abeq.org.br/bjche

Vol. 31, No. 02, pp. 531 - 542, April - June, 2014

dx.doi.org/10.1590/0104-6632.20140312s00001590

\title{
SIMPLE MULTICOMPONENT BATCH DISTILLATION PROCEDURE WITH A VARIABLE REFLUX POLICY
}

\author{
A. N. García ${ }^{1 *}$, J. C. Z. Loría ${ }^{2}$, A. R. Marín ${ }^{2}$ and A. V. C. Quiroz ${ }^{2}$ \\ ${ }^{1}$ Universidad Autónoma de Yucatán, Facultad de Ingeniería Química, Phone: + 52 (999) 9460956, \\ Campus de Ingenierías y Ciencias Exactas, Periférico Norte Kilómetro 33.5, Tablaje Catastral 13615, \\ Colonia Chuburna de Hidalgo Inn, C.P. 97203, Mérida, Yucatán, México. \\ E-mail: narvaez1410@hotmail.com \\ ${ }^{2}$ Universidad Autónoma del Carmen, Dependencia Académica de Ciencias Químicas y Petrolera (DACQyP), \\ Facultad de Química, Phone: + 52 (938) 3828484, Campus Principal, Calle 56 No. 4 Esquina Avenida Concordia, \\ Colonia Benito Juárez, C.P. 24180, Cd. del Carmen, Campeche, México.
}

(Submitted: April 29, 2013 ; Revised: July 3, 2013 ; Accepted: July 10, 2013)

\begin{abstract}
This paper describes a shortcut procedure for batch distillation simulation with a variable reflux policy. The procedure starts from a shortcut method developed by Sundaram and Evans in 1993 and uses an iterative cycle to calculate the reflux ratio at each moment. The functional relationship between the concentrations at the bottom and the dome is evaluated using the Fenske equation and is complemented with the equations proposed by Underwood and Gilliland. The results of this procedure are consistent with those obtained using a fast method widely validated in the relevant literature.

Keywords: Batch distillation; Variable reflux policy; Shortcut procedure.
\end{abstract}

\section{INTRODUCTION}

Mathematical modeling of batch distillation is more complex than the modeling of continuous distillation because the process is dynamic and its behavior is represented by a system of differential algebraic equations (DAE). The complexity further increases when multicomponent mixtures, columns with a great number of plates, column hydraulics, and the system thermodynamics are considered.

To solve this problem, rigorous mathematical models are currently of great interest because computers are very easy to use, have high accuracy, and possess great processing capabilities. However, the use of equipment such as tablets or laptops with reduced data-processing capacity necessitates a search for simple methods that can predict the behavior of chemical processes without using higher processing capacities. In addition, this kind of method will always be necessary to provide the initial data for mathematical optimization processes or as a first approximation to the behavior of distillation techniques.

An acceptable approach to modeling the behavior of a batch distillation process has been the use of the simplified or shortcut methods proposed by authors such as Diwekar (1988), Sundaram and Evans (1993), Zamar et al. (1998), Barolo and Guarise (1999), and Ehsani (2002), among others.

Both the work by Diwekar and that by Sundaram and Evans have been widely discussed and validated and are incontrovertible references in the modeling, simulation, and optimization of the batch distillation process. The first author considered constant reflux and variable reflux policies in their work, while the latter authors only proposed a constant reflux policy.

The difference between the two works lies in the way in which the functional relationship between the compositions at the dome and the bottom are

*To whom correspondence should be addressed 
addressed. Diwekar (1988) proposed the use of the Hensgtebeck-Geddes equation for calculating the minimum number of equilibrium stages $\left(N_{\min }\right)$, which is a modification of Fenske's equation, while the work by Sundaram and Evans (1993) used the original equation of Fenske.

For the relationship between the minimum number of equilibrium stages $\left(N_{\min }\right)$ and the minimum reflux ratio $\left(R_{\min }\right)$, both studies used the equations of Underwood and Gilliland. However, the work by Sundaram and Evans considers mixtures whose behavior is classified as Class I (all components are distributed along the column). Since $N_{\min }$ and $R_{\min }$ are limit conditions necessary for the design of distillation columns, shortcut methods are useful in the design process, and they are also suitable for simulation and mathematical optimization.

In practice, the work by Sundaram and Evans (1993) is easier to apply because of the simplifications made by the authors. However, because this work does not consider a variable reflux policy, the Diwekar (1988) proposal has actually been more widely applied.

To address this situation, this paper incorporates the concepts developed by Sundaram and Evans (1993) and presents a procedure that takes into account a variable reflux policy. First, the procedure uses the mathematical model developed by Sundaram and Evans (1993) for a constant reflux policy, which includes a global balance of matter, partial balances of matter, and a functional relationship for the component concentrations at the dome and the bottom. The model presented by Sundaram and Evans (1993) is as follows:

Global balance:

$B_{\text {new }}=B_{\text {old }}-\left(\frac{V}{1+R}\right) \Delta t$

Partial balances:

$x_{B, \text { new }}^{(i)}=x_{B, \text { old }}^{(i)}+\left[x_{D}^{(i)}-x_{B}^{(i)}\right]_{\text {old }}\left[\frac{B_{\text {new }}-B_{\text {old }}}{B_{\text {old }}}\right]$

Fenske's equation:

$$
N_{\min }=\frac{\log \left\{\left[\frac{x_{D}^{(i)}}{x_{B}^{(i)}}\right]\left[\frac{x_{B}^{(r)}}{x_{D}^{(r)}}\right]\right\}}{\log \alpha_{i, r}}
$$

$$
\begin{aligned}
& x_{D}^{(i)}=x_{B}^{(i)}\left[\frac{x_{D}^{(r)}}{x_{B}^{(r)}}\right] \alpha_{i, r}^{N_{\min }} \\
& x_{D}^{(r)}=\frac{x_{B}^{(r)}}{\sum_{i=1}^{n c} x_{B}^{(i)} \alpha_{i, r}^{N_{\min }}}
\end{aligned}
$$

Gilliland's correlation:

$$
\begin{aligned}
& Y=1-\exp \left[\frac{(1+54.4 X)(X-1)}{(11+117.2 X) \sqrt{X}}\right] \\
& Y=\frac{N-N_{\min }}{N+1} \\
& X=\frac{R-R_{\min }}{R+1}
\end{aligned}
$$

Eduljee's correlation:

$$
\frac{N-N_{\min }}{N+1}=0.75\left[1-\left(\frac{R-R_{\min }}{R+1}\right)^{0.5668}\right]
$$

Underwood equation (Class I):

$$
R_{\min }=\frac{\alpha_{1, r}^{N_{\min }}-\alpha_{1, r}}{\left(\alpha_{1, r}-1\right) \sum_{i=1}^{n c} x_{B i} \alpha_{1, r}^{N_{\min }}}
$$

Underwood equations (Class II):

$$
\sum_{i=1}^{n c} \frac{\alpha_{i, r} x_{B}^{(i)}}{\alpha_{i, r}-\phi}=1-q
$$

$$
R_{\text {min }}+1=\sum_{i=1}^{c} \frac{\alpha_{i, r} x_{D}^{(i)}}{\alpha_{i, r}-\phi}
$$

where $q$ is the feed condition defined as the ratio of the heat required to vaporize $1 \mathrm{~mol}$ of the feed to the molar latent heat of the feed. If the feed is at its boiling point, then $q=1$.

Our proposal is based on the idea that, to maintain a product with the desired concentration, it is necessary to iteratively find the reflux ratio. That is, if one assumes that, at any given time, there is a 
constant reflux ratio that will achieve the desired concentration of the product, then it is possible to use a shortcut method, as developed by Sundaram and Evans (1993), since their results will be valid.

\section{REFLUX POLICIES}

The distillation columns can work with the following policies: 1) constant reflux, 2) variable reflux, and 3) optimal reflux. The two other existing policies are the total reflux policy and the zero reflux policy.

When the constant reflux policy is used, the concentrations of the components in the dome vary at each instant, so an average concentration in the product is considered for the desired component $i$.

In the case of variable reflux, the composition of the desired component does not vary with time, i.e., it remains constant during the operation of the column. In this case, the other components of the product may vary or remain constant, depending on the mathematical model of the process.

Finding the optimal reflux policy requires the use of some mathematical optimization method to learn about the optimal profile of the control variable (reflux ratio). The aim of this operation is to solve the optimal control problems to obtain the maximum amount of distillate, the shortest separation time for the mixture, the biggest gain, or the lowest consumption of energy, among other goals.

The total reflux policy is used to stabilize the separation process. With this policy, no product is obtained in the dome of the column.

A zero reflux policy is used to exhaust some of the components so as to reduce a high concentration to the desired concentration in less time. We found that this policy was used in certain stages of the separation process when a variable reflux ratio was used.

\section{SHORTCUT MATHEMATICAL MODEL}

As mentioned above, Equations (1) to (12) present the mathematical model developed by Sundaram and Evans (1993). Equation (1) enforces the overall balance of matter at each moment, considering the changes that occur at the bottom and the dome.

Equation (2) is an instantaneous partial balance for component $i$ considering Equation (1). Equations (3) and (4) are known as Fenske's equation. Equation (5) is obtained by applying a sum over all components of the mixture $(i=1,2, \ldots, n c)$ and solving for the reference component $(r)$, i.e.,

$$
\begin{aligned}
& x_{D}^{(i)}=x_{B}^{(i)}\left[\frac{x_{D}^{(r)}}{x_{B}^{(r)}}\right] \alpha_{i, r}{ }^{N_{\min }} ; i=1,2, \ldots, n \\
& \sum_{i=1}^{n c} x_{D}^{(i)}=\left[\frac{x_{D}^{(r)}}{x_{B}^{(r)}}\right] \sum_{i=1}^{n c}\left[x_{B}^{(i)} \alpha_{i, r}{ }^{N_{\min }}\right]=1 \\
& x_{D}^{(r)}=\frac{x_{B}^{(r)}}{\sum_{i=1}^{n c}\left[x_{B}^{(i)} \alpha_{i, r}{ }^{N_{\min }}\right]}
\end{aligned}
$$

Equation (6) is Gilliland's correlation, and Equation (9) is an acceptable simplification of Equation (6), known as Eduljee's correlation. Equations (7) and (8) are the equations used in Gilliland's and Eduljee's correlations. Equations (10), (11), and (12) were developed by Underwood.

To select the light and heavy key components, we considered the concepts used by Sundaram and Evans (1993). In this framework, the light key component $(l k)$ is defined as one that is present in the residue in important amounts and the heavy key component $(h k)$ is defined as one that is present in the distillate in important amounts. In the mathematical model represented by Equations (1) to (12), the authors consider a constant reflux policy. Therefore, as mentioned previously, to extend this mathematical model to other reflux policies, it is necessary to consider an iterative process that compares the value of the concentration of the light key component $(l k)$ at the dome with its expected concentration.

The variable to be adjusted to ensure that both the expected and calculated concentrations are equal is the reflux ratio $\left(R_{t}\right)$. Therefore, it is necessary to obtain an expression in terms of $R_{t}$ for the component whose concentration is constant in the dome (lk), as in Equation (4):

$$
x_{D}^{(l k)}=x_{B}^{(l k)}\left[\frac{x_{D}^{(r)}}{x_{B}^{(r)}}\right] \alpha_{l k, r}^{N_{\min }}
$$

This expression can be rearranged so that it is expressed as an equality to zero:

$$
f\left(R_{t}\right)=\left[\frac{x_{D}^{(r)}}{x_{B}^{(r)}}\right] \alpha_{l k, r}^{N_{\min }}-\left[\frac{x_{D}^{(l k)}}{x_{B}^{(l k)}}\right]=0
$$

The iterative process can be performed using the Newton-Raphson method, for which it is necessary to find the derivative of Equation (16): 


$$
f^{\prime}\left(R_{t}\right)=\left[\frac{x_{D}^{(r)}}{x_{B}^{(r)}}\right] \alpha_{l k, r}^{N_{\min }} \ln \left(\alpha_{l k, r}\right)-\left(\frac{d N_{\min }}{d R_{t}}\right)
$$

where (using Eduljee's correlation)

$$
\left(\frac{d N_{\min }}{d R_{t}}\right)=0.4251(N+1)\left(\frac{R_{t}+1}{R_{t}-R_{\min }}\right)^{0.4332}\left[\frac{R_{\min }+1}{\left(R_{t}+1\right)^{2}}\right]
$$

and the approach to $R_{t}$ for each iteration, is given by

$$
R_{t}=R_{t}-\frac{f\left(R_{t}\right)}{f^{\prime}\left(R_{t}\right)}
$$

\section{THE ALGORITHM}

The algorithm for solving the system of equations making up the proposed procedure starts by assuming the value of the reflux ratio, the minimum number of equilibrium stages, and the minimum reflux ratio.

In an outer loop, the reflux ratio is calculated with Equation (19), and the value obtained is compared with the assumed value. The cycle (loop) is stopped when the difference between the values is less than the error $\left[\operatorname{abs}\left(R_{t}-R_{\text {assumed }}\right) \leq\right.$ error]. There is also an inner loop that calculates the minimum number of stages and the minimum reflux ratio. Figure 1 shows the sequence of steps for the proposed procedure.

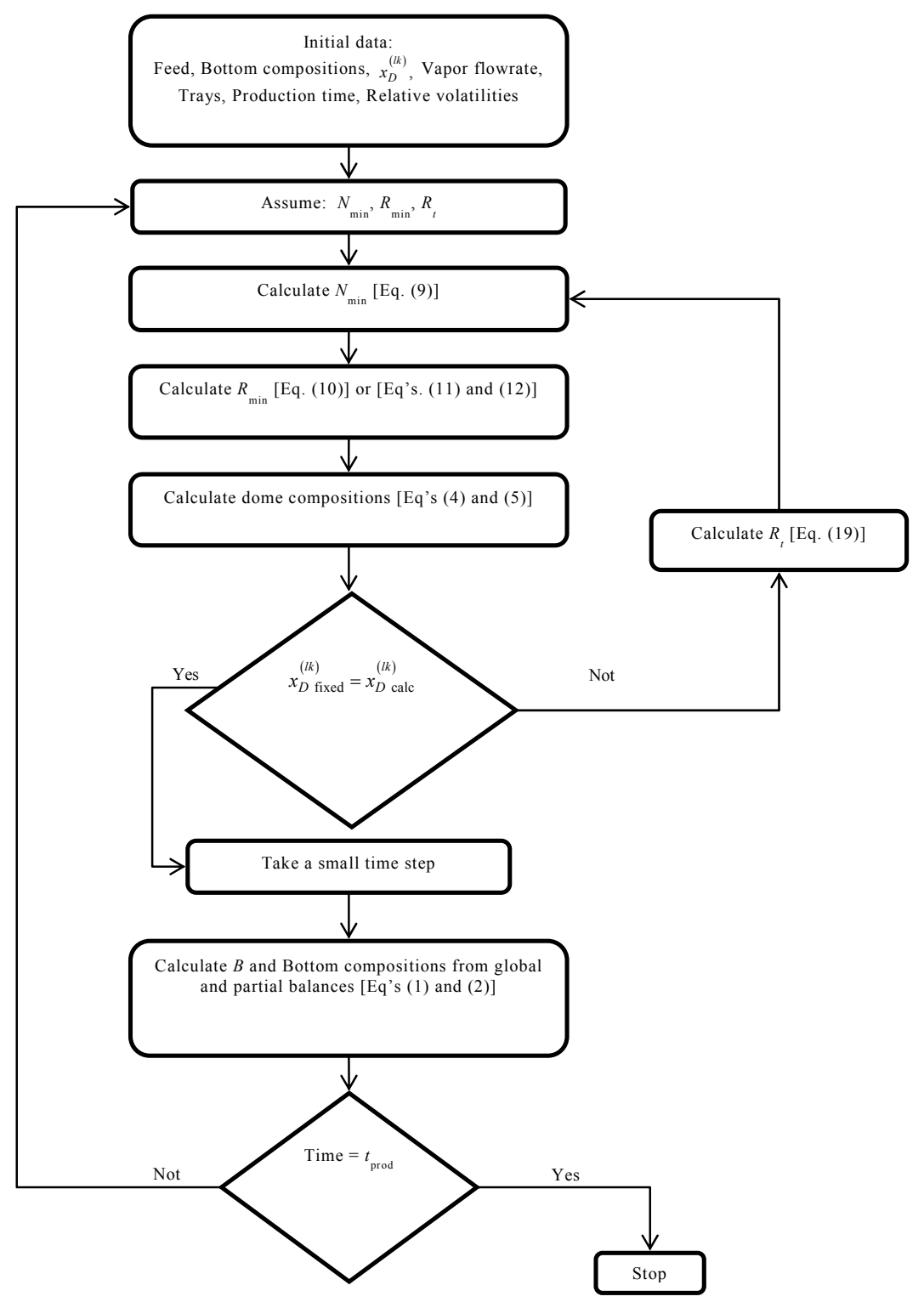

Figure 1: Shortcut model algorithm (procedure proposed). 


\section{MODEL TESTING, RESULTS, AND VALIDATION}

The proposed procedure has been evaluated by solving the equations in the mathematical model for several multicomponent mixtures. The results were compared with the results obtained using the shortcut method developed by Diwekar (1988), which is included in the MultibatchDS software (Diwekar and Madhavan, 1991). This reference shortcut method has been extensively validated in the literature. The proposed procedure has been tested with several case studies, but this work only presents the results for ten mixtures.

For our solution, we considered a selected reference component $(r)$ to be the heavy key component $(h k)$ and started the procurement process when the expected concentration of the light key component (lk) was obtained. In other words, the total reflux condition is not used, so the stable state is not reached.

If the intention were to start the procurement process from the stable state, it would be necessary first to carry out the following two stages in the process: 1) take the process to the steady state using a total reflux policy until the column conditions do not vary, 2) obtain the product at zero reflux until the desired concentration of the light key component is reached, and 3) obtain the product at variable reflux until a desired time.

For steps 1 and 2 in Fig. 1, the shortcut method is applied, as described by Sundaram and Evans (1993). In the first stage, the minimum number of plates equals the total number of plates $\left(N_{\min }=N\right)$, and in the second stage, the minimum reflux ratio is zero (since negative $R_{\min }$ values are obtained when $R_{t}$ is zero).
Table 1 presents the data for each case study.

The data on mixtures 1, 2, 6, and 7 were taken from the work of Sundaram and Evans (1993). For mixture 3, data were obtained from Diwekar and Madhavan (1991), and for mixture 4, data were obtained from Seader and Henley (1998). The mixture 5 data were taken from Mujtaba (2004). Data for mixtures 8 and 10 were taken from Luyben (1971). The mixture 9 data were taken from Dechema (1987). All data were used by their respective authors for simulation of the batch distillation process using a constant reflux ratio policy.

Then, for systems in which all components are distributed throughout the column (Class I mixture: "separations such that, with infinite plates, all components of the feed are present in both the top product and bottom product," Shiras et al., 1950), Equation (10) is used. When the components are not distributed (Class II mixture: "separations such that, with infinite plates, some of the components are completely in the top product or completely in the bottom product," Shiras et al., 1950), Equations (11) and (12) are used. However, in this case it is necessary to calculate the Underwood parameter $(\phi)$, whose value is between the relative volatility of the light component $(l k)$ and that of the heavy component $(h k)$.

This work considers Class I binary mixtures and both Class I and Class II ternary and multicomponent mixtures. In each case, the results have been compared with those obtained using the shortcut method

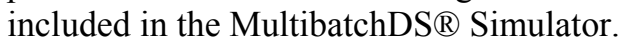

In particular, we have used Equation (10) for mixtures 1, 2, 4, 8, 9, and 10 and Equations (11) and (12) for the others. The results of the simulations are presented in the following figures.

Table 1: Starting conditions for the solution obtained using the shortcut variable reflux method.

\begin{tabular}{|c|c|c|c|c|c|c|c|c|c|c|c|c|c|c|c|}
\hline \multirow{2}{*}{ Mixture } & \multicolumn{4}{|c|}{ Molar fraction feed } & \multicolumn{4}{|c|}{ Relative volatilities } & \multirow{2}{*}{$N^{+}$} & \multirow{2}{*}{$r$} & \multirow{2}{*}{$x_{D}^{(l k)}$} & \multirow{2}{*}{$\boldsymbol{V}$} & \multirow{2}{*}{$\boldsymbol{F}$} & \multirow{2}{*}{$t_{\text {prod }}$} & \multirow{2}{*}{ Class } \\
\hline & 1 & 2 & 3 & 4 & 1 & 2 & 3 & 4 & & & & & & & \\
\hline 1 & 0.250 & 0.250 & 0.250 & 0.25 & 2.00 & 1.50 & 1.20 & 1.00 & 5 & 4 & 0.70 & 120.0 & 100.0 & 1.0 & $\mathrm{I}$ \\
\hline 2 & 0.100 & 0.300 & 0.100 & 0.50 & 2.00 & 1.50 & 1.20 & 1.00 & 30 & 4 & 0.95 & 120.0 & 800.0 & 2.0 & I \\
\hline 3 & 0.300 & 0.200 & 0.450 & 0.05 & 2.00 & 1.50 & 1.00 & 0.50 & 10 & 3 & 0.80 & 100.0 & 150.0 & 2.0 & II \\
\hline 4 & 0.330 & 0.330 & 0.340 & - & 2.00 & 1.50 & 1.00 & - & 3 & 3 & 0.58 & 110.0 & 100.0 & 2.0 & $\mathrm{I}$ \\
\hline 5 & 0.407 & 0.394 & 0.199 & - & 2.61 & 1.48 & 1.00 & - & 10 & 3 & 0.99 & 50.0 & 200.0 & 2.0 & II \\
\hline 6 & 0.400 & 0.500 & 0.100 & - & 2.00 & 1.50 & 1.00 & - & 20 & 3 & 0.95 & 150.0 & 250.0 & 2.0 & II \\
\hline 7 & 0.330 & 0.330 & 0.340 & - & 6.00 & 2.00 & 1.00 & - & 4 & 3 & 0.98 & 100.0 & 150.0 & 3.0 & II \\
\hline 8 & 0.490 & 0.510 & - & - & 2.00 & 1.00 & - & - & 9 & 2 & 0.95 & 220.0 & 500.0 & 2.5 & $\mathrm{I}$ \\
\hline 9 & 0.200 & 0.800 & - & - & 3.50 & 1.00 & - & - & 5 & 2 & 0.80 & 100.0 & 1000.0 & 2.0 & $\mathrm{I}$ \\
\hline 10 & 0.100 & 0.900 & - & - & 3.00 & 1.00 & - & - & 20 & 2 & 0.98 & 100.0 & 200.0 & 1.0 & I \\
\hline
\end{tabular}

Units: $t_{\text {prod }}(\mathrm{h}) ; F(\mathrm{kmol} / \mathrm{h}) ; V(\mathrm{kmol} / \mathrm{h}) .{ }^{+}$The reboiler must be considered. 


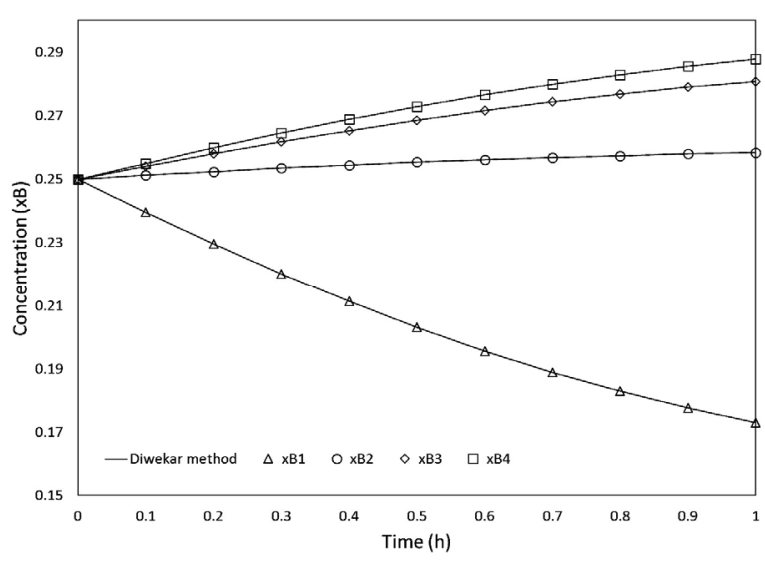

(a) Bottom concentration profiles.

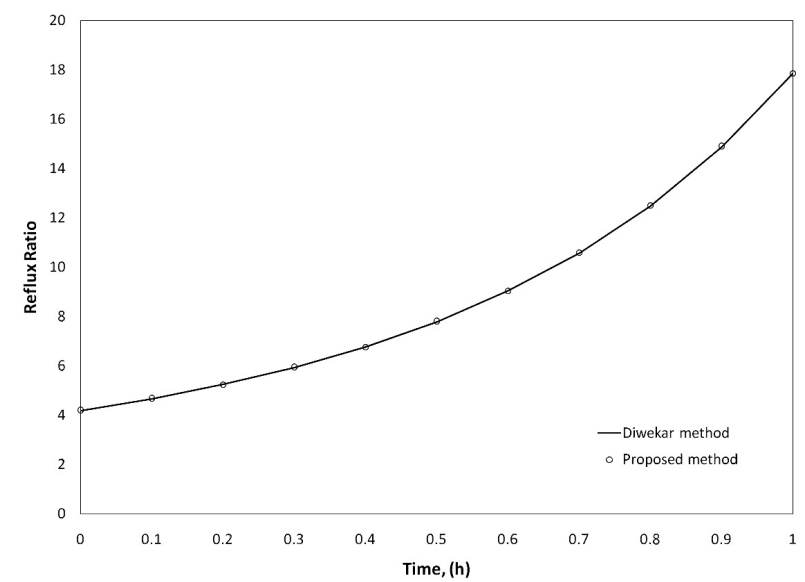

(b) Reflux ratio profiles.

Figure 2: Mixture 1: Comparison of the proposed shortcut method with the Diwekar shortcut method.

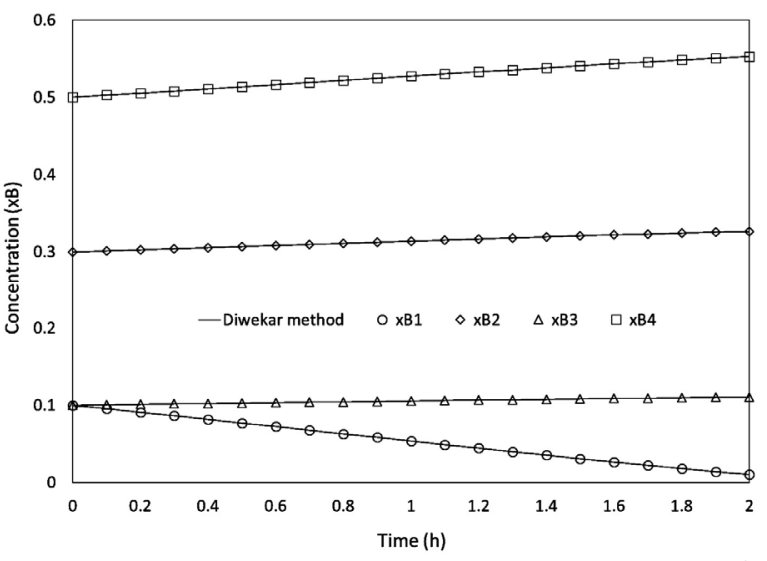

(a) Bottom concentration profiles.

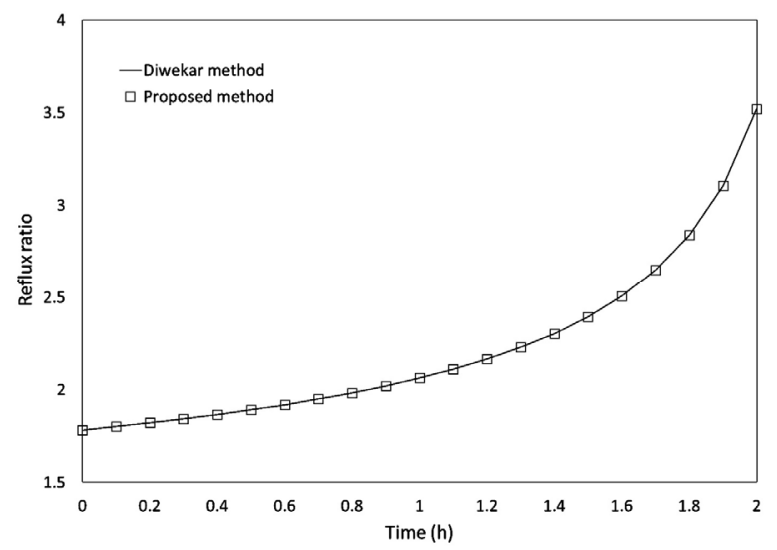

(b) Reflux ratio profiles.

Figure 3: Mixture 2: Comparison of the proposed shortcut method with the Diwekar shortcut method.

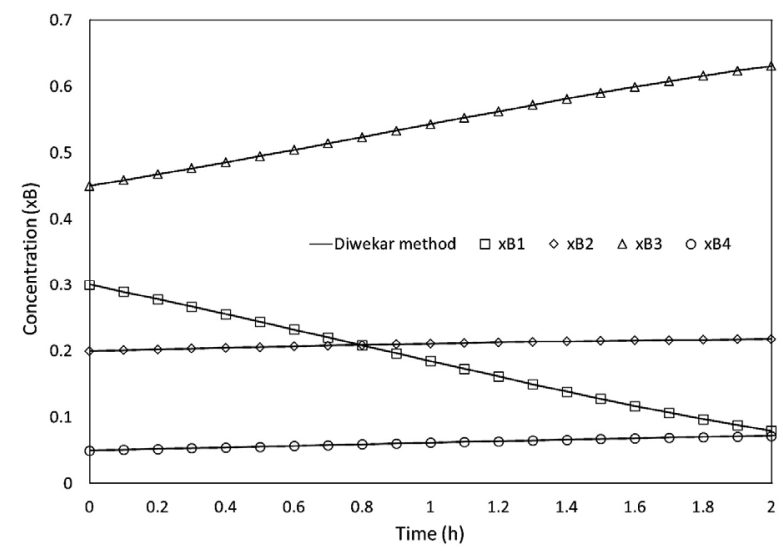

(a) Bottom concentration profiles.

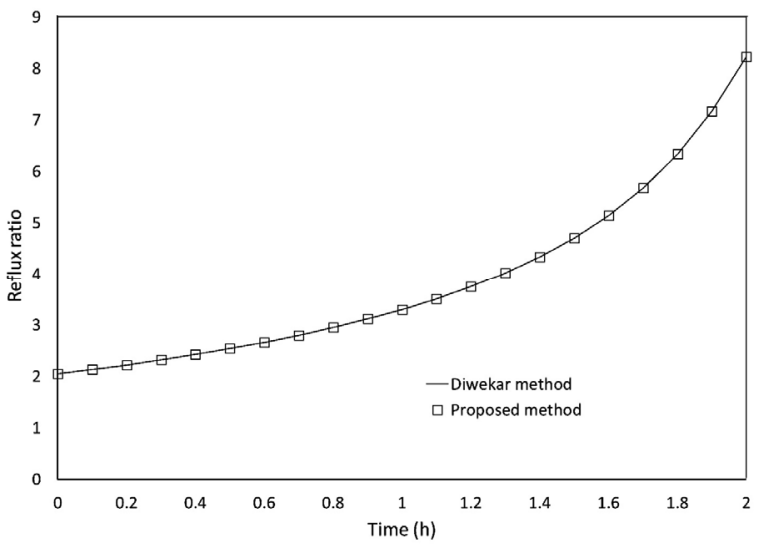

(b) Reflux ratio profiles.

Figure 4: Mixture 3: Comparison of the proposed shortcut method with the Diwekar shortcut method. 


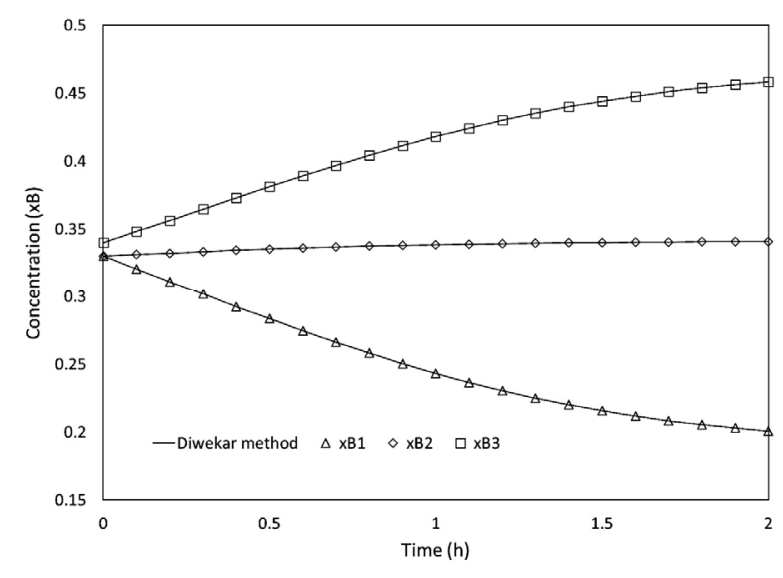

(a) Bottom concentration profiles.

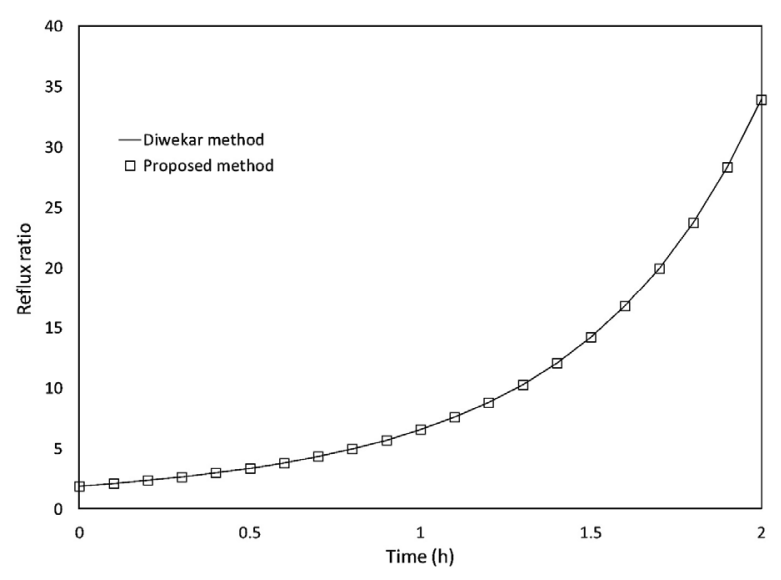

(b) Reflux ratio profiles.

Figure 5: Mixture 4: Comparison of the proposed shortcut method with the Diwekar shortcut method.

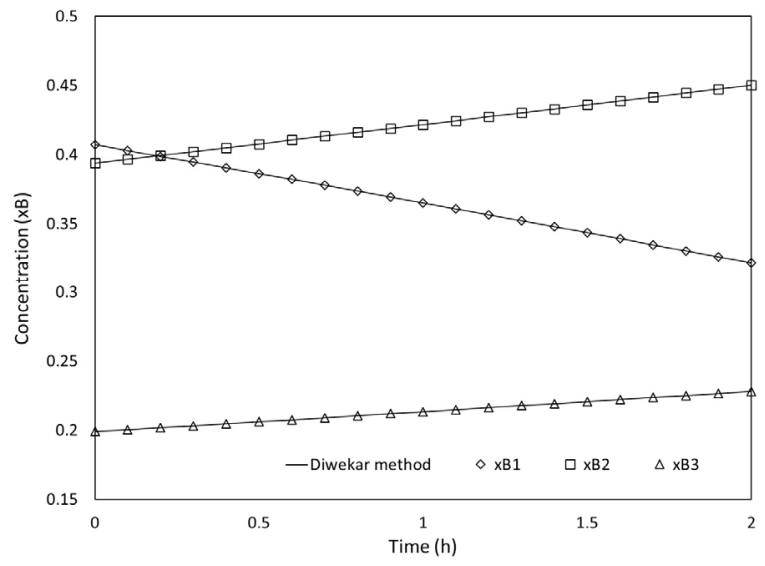

(a) Bottom concentration profiles.

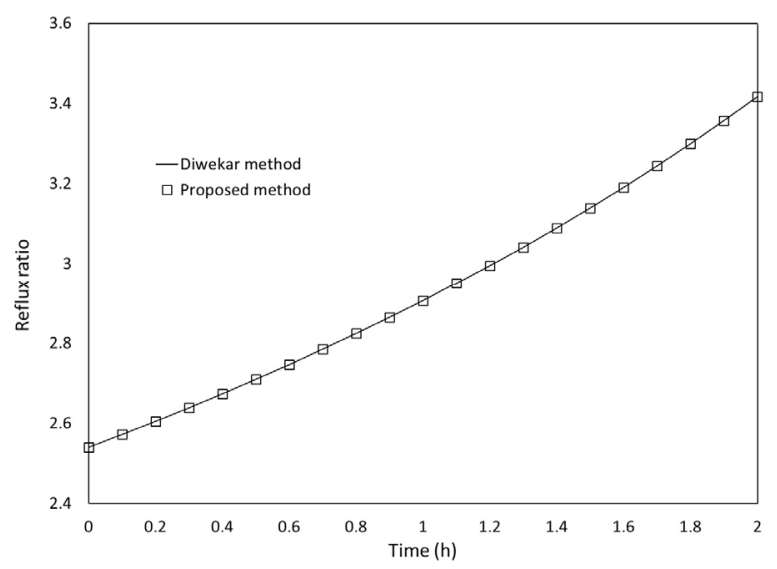

(b) Reflux ratio profiles.

Figure 6: Mixture 5: Comparison of the proposed shortcut method with the Diwekar shortcut method.

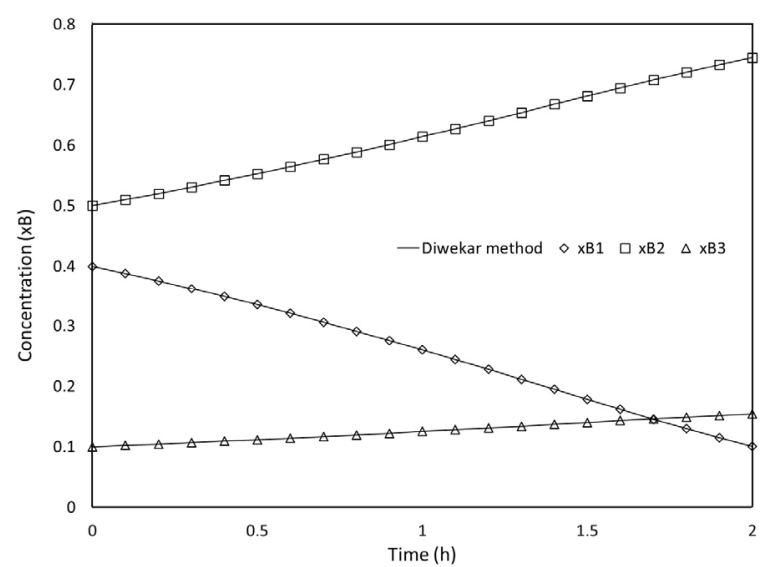

(a) Bottom concentration profiles.

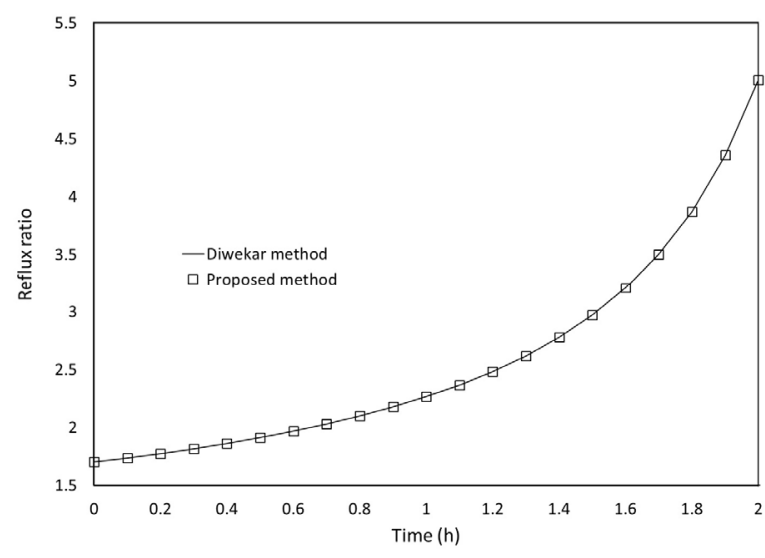

(b) Reflux ratio profiles.

Figure 7: Mixture 6: Comparison of the proposed shortcut method with the Diwekar shortcut method. 


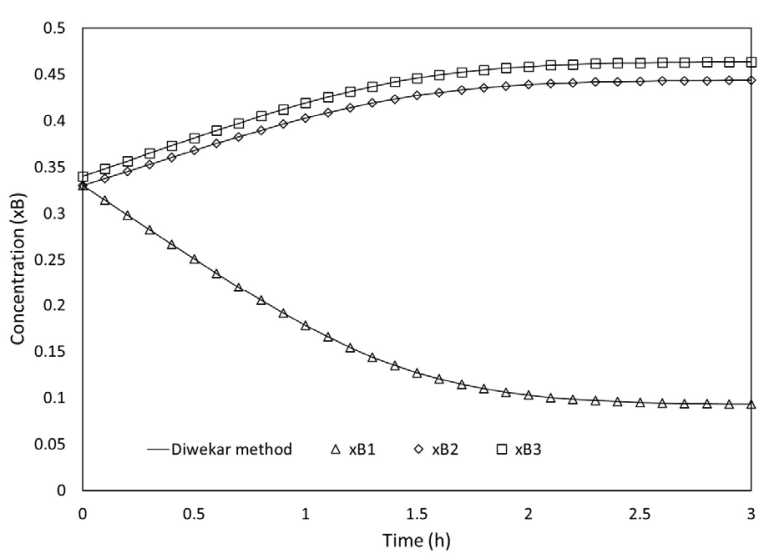

(a) Bottom concentration profiles.

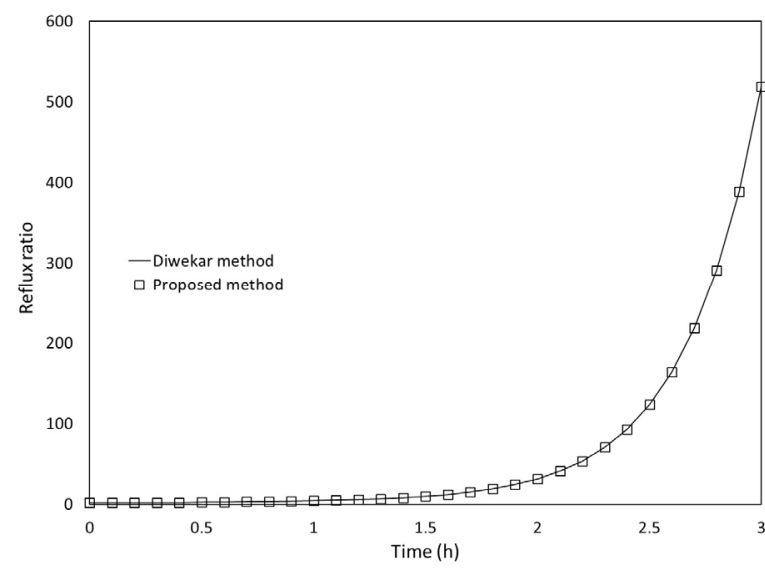

(b) Reflux ratio profiles.

Figure 8: Mixture 7: Comparison of the proposed shortcut method with the Diwekar shortcut method.

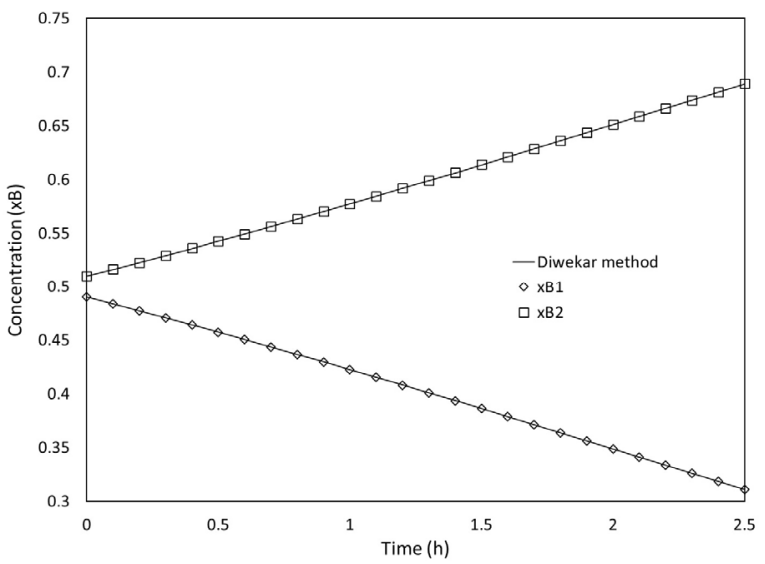

(a) Bottom concentration profiles.

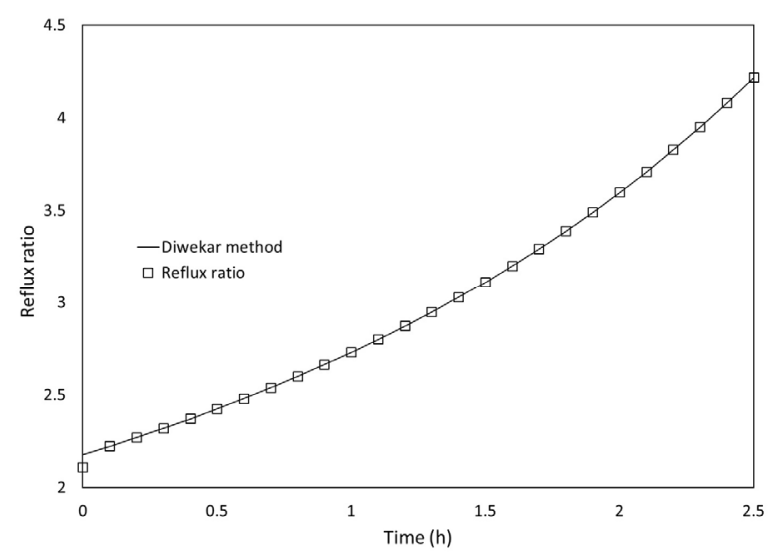

(b) Reflux ratio profiles.

Figure 9: Mixture 8: Comparison of the proposed shortcut method with the Diwekar shortcut method.

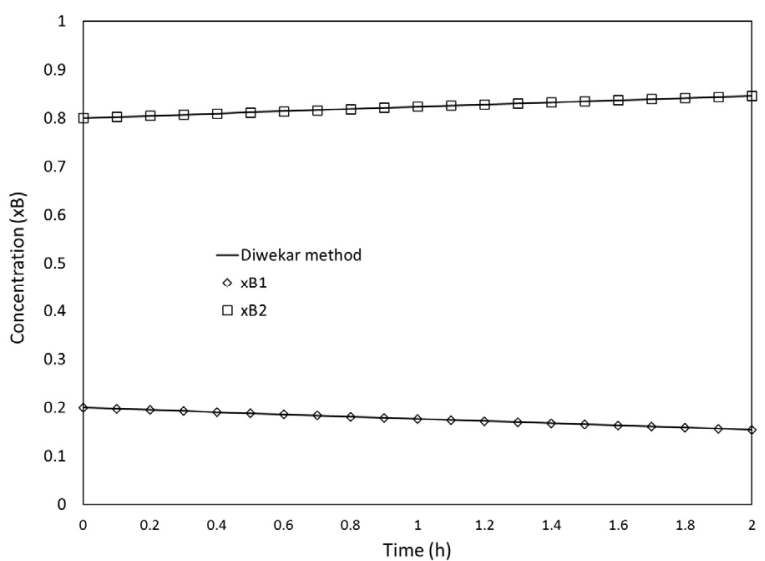

(a) Bottom concentration profiles.

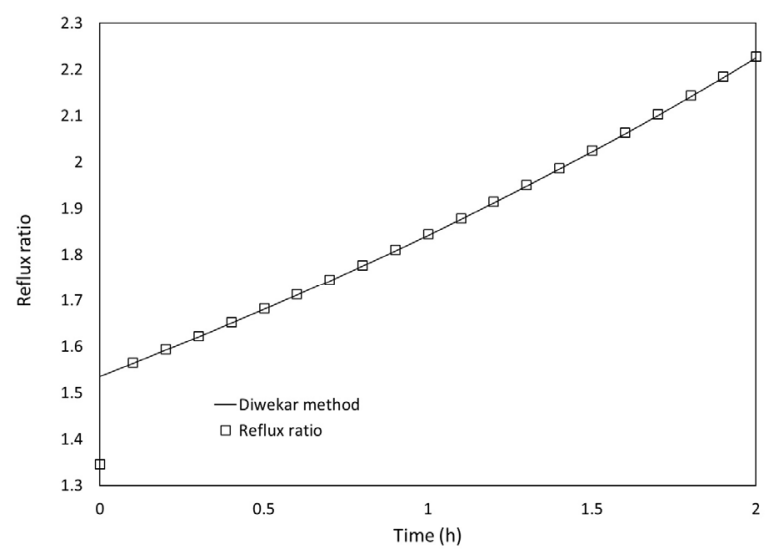

(b) Reflux ratio profiles.

Figure 10: Mixture 9: Comparison of the proposed shortcut method with the Diwekar shortcut method. 


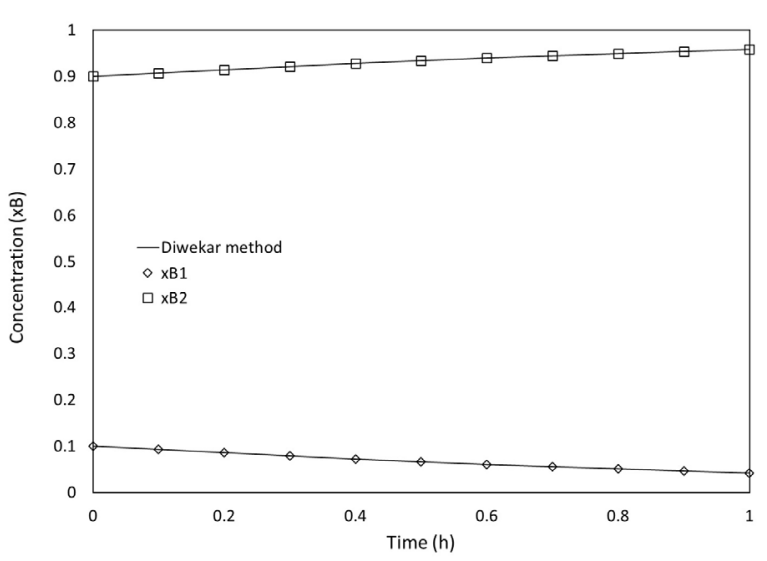

(a) Bottom concentration profiles.

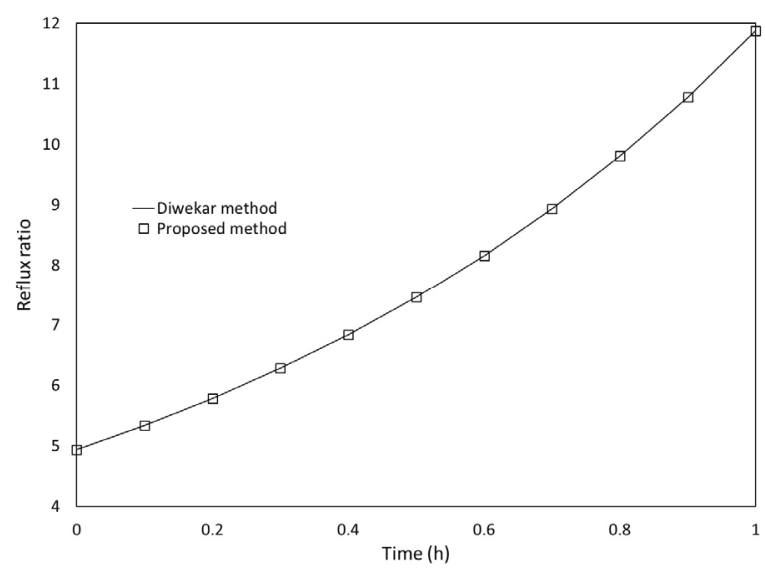

(b) Reflux ratio profiles.

Figure 11: Mixture 10: Comparison of the proposed shortcut method with the Diwekar shortcut method.

Figures 2-11 all show that the behavior of the concentration profiles in the bottom of the column is as expected, i.e., the more volatile component becomes depleted and the heavier components are enriched. Of course, the more volatile components will be exhausted within a certain time. In this work, the most volatile component is regarded as the light key component. When this component is exhausted, the next light key component must be chosen to be separated in the next cut, and so on, until each component is obtained as a product, with the lightest components obtained at the dome and the heaviest at the bottom.

Each of the figures (Figs. 2(b)-12(b)) shows a similar profile for the reflux ratio, which is characteristic of the variable reflux process. In particular, the reflux ratio increases as time passes until the concentration of the desired product is reached. As expected, when the concentration of the more volatile component is greater, a smaller amount of reflux is required to achieve the desired concentration in the dome. As the most volatile component becomes depleted, the amount of reflux required to achieve the desired concentration is higher, so a lot of reflux might be required to achieve the desired concentration of the light key component. A large amount of reflux means that the amount of product obtained is very small. In this case, energy costs can become significant with no benefit to the process, because most of the energy supplied to the reboiler is withdrawn from the condenser during the distillation.

For example, in Figure 8 (mixture 7), we can see that it would be appropriate to make a cut in the production at a time of 1.5 hours because, beyond this time, the changes in the reflux ratio profile are almost of exponential order, and the product is obtained in the desired concentration but only in very small quantities. One factor that causes the need for relatively large amounts of reflux is that a small number of plates have been used in the process to obtain a high concentration of the desired product. Because the lighter component is more volatile than the other components, the initial separation is easy and produces a greater amount of product. However, as the process proceeds, the lighter component is exhausted quickly, and the residual amounts of the component require a greater amount of reflux to separate, resulting in very small quantities of the desired product. In fact, the use of very high amounts of reflux in this case (mixture 7) has an academic purpose only, to illustrate the mathematical solution of the proposed model. The energy costs are not justified by the additional product obtained unless the product is quite valuable so that the process has no economic loss.

For each mixture, the results for the concentrations of the components in the bottom of the column and the profiles of the reflux ratio obtained using the proposed procedure are similar to the results of the shortcut method proposed by Diwekar (1988). There were no significant deviations in the respective values, but the current work does need adequate initial values for the reflux ratio $\left(R_{t}\right)$, since the values chosen directly affect the speed of convergence and the possibility of an infinite loop.

The proposed method is an approximate one, and there are conditions for which it is not suitable. Therefore, it is necessary to reiterate that this work assumes that the batch column can be properly represented using the Fenske-Underwood-Gilliland equa- 
tions, that there is a thermodynamic equilibrium at every time step, and that the effect of holdup on the dynamic behavior of the batch column can be neglected. In addition, this study does not consider the separation of azeotropic mixtures, because the modeling and simulation of this type of mixture requires modification of the proposed method, which is not within the scope of this work.

Whereas the original method of Sundaram and Evans considered only a constant relative volatility and Class I mixtures, this work also considers Class II mixtures and the possibility of variations in the relative volatilities.

When there is variation in the relative volatilities along the column and with time, the average values can be obtained as:

$\left(\alpha_{i, j}\right)_{\text {average }}=\sqrt{\left(\alpha_{i, j}\right)_{B}\left(\alpha_{i, j}\right)_{D}}$

where

$$
\alpha_{i, j}=\frac{K_{i}}{K_{j}}
$$

and

$K_{i}=\frac{y^{(i)}}{x^{(i)}}$

Using Antoine's equation to calculate the vaporliquid equilibrium constant $\left(K_{i}\right)$ :

$$
K_{i}=\frac{1}{P} \exp \left[A_{i}-\frac{B_{i}}{T+C_{i}}\right]
$$

where $T$ is the temperature of bubble and $P$ is the process operating pressure.

Now, if in addition to the temperature, the relative volatility directly influences the concentration of the components of the mixture, a modification of Raoult's Law can be used, i.e.,

$$
K_{i}=\left(\frac{P_{i}^{\text {vap }}}{P}\right) \gamma_{i}=\left\{\frac{1}{P} \exp \left[A_{i}-\frac{B_{i}}{T+C_{i}}\right]\right\} \gamma_{i}
$$

where $\gamma$ is the activity coefficient. In this work, we used Wilson's equation to calculate this coefficient.

Figure 12 shows the results obtained considering variations in the relative volatilities for a mixture during the separation process where $K$ is only temperature dependent. The data used were a binary equimolar cyclohexane-toluene mixture (2.42 initial relative volatility), 6 theoretical plates, 100 moles of feed, a $120 \mathrm{~mol} / \mathrm{h}$ vapor rate $(V)$, a product with $99 \%$ cyclohexane, and a time of production of 3 hours.

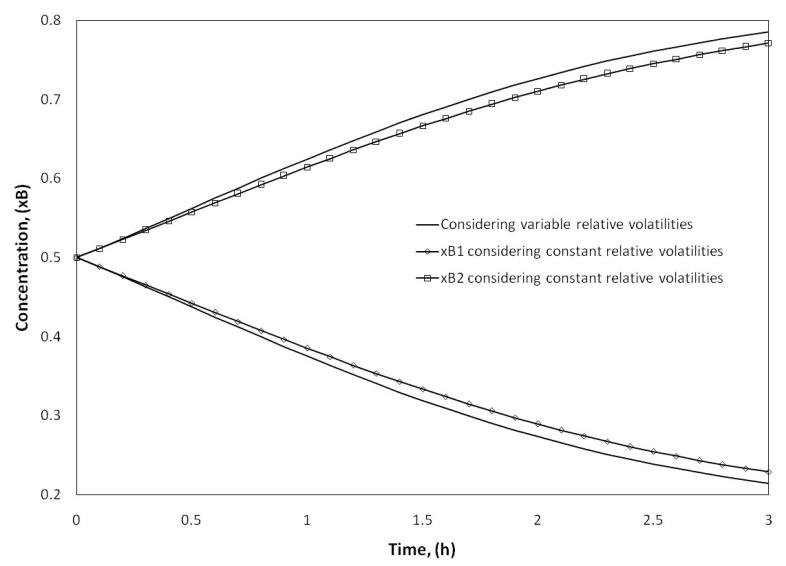

(a) Bottom concentration profiles.

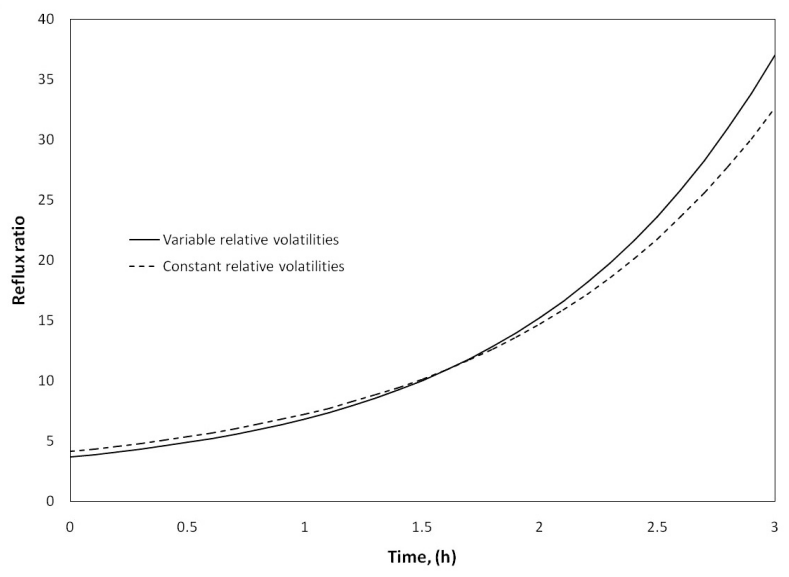

(b) Reflux ratio profiles.

Figure 12: Comparison of concentrations and reflux ratios considering constant and variable relative volatilities.

The results showed a variation of up to $6 \%$ with respect to the bottom concentration and up to $13.4 \%$ with respect to the reflux ratio. These variations are due to the increase of approximately 10 degrees $\left(T_{0}=92.72{ }^{\circ} \mathrm{C} ; T_{f}=101.97{ }^{\circ} \mathrm{C}\right)$ in temperature at the bottom due to the depletion of the more volatile component and to the enrichment of the heavier component.

Figure 13 shows the results obtained considering variations in the relative volatilities for a mixture during the separation process where $K$ was obtained 
using Equation (24). The data used were a benzene (0.4274)/2-methoxyethanol (0.5726) mixture (5.92 initial relative volatility), 10 theoretical plates, 200 moles of feed, a $120 \mathrm{~mol} / \mathrm{h}$ vapor rate $(V)$, a product with $90.58 \%$ benzene, and a time of production of 2 hours.

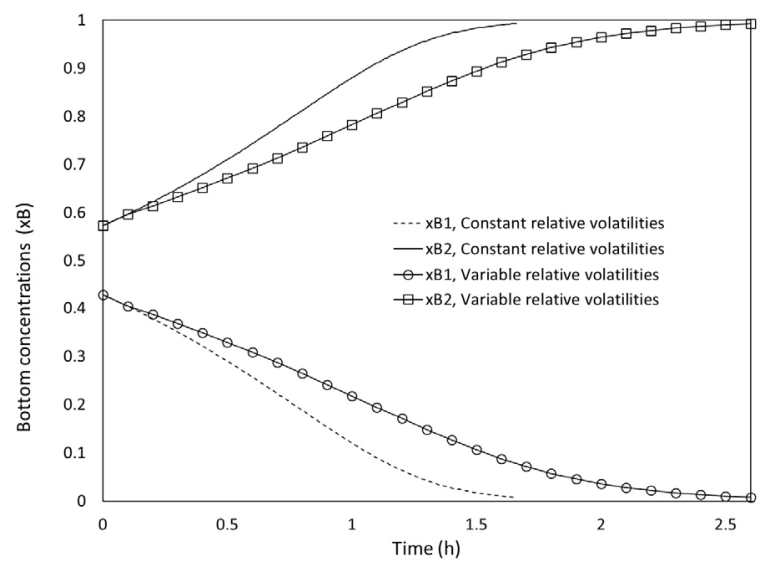

(a) Bottom concentration profiles.

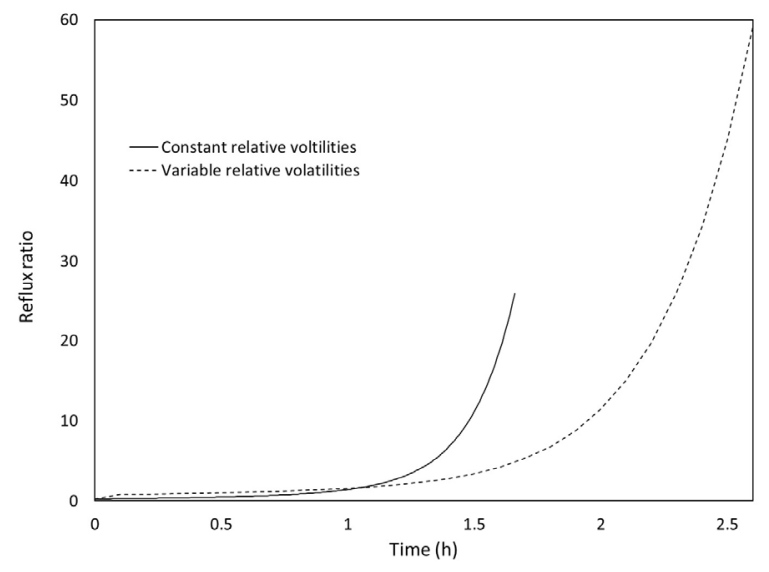

(b) Reflux ratio profiles.

Figure 13: Comparison of concentrations and reflux ratios obtained considering constant and variable relative volatilities.

This figure shows the influence of both temperature and concentration on the separation process. Here, the time to reach the same percentage of exhaustion is smaller for the process with constant relative volatility, because the separation of the mixture is easier at higher $\alpha$ values and, of course, requires less reflux to achieve the desired concentration. In this case, the impact of temperature and concentration on the relative volatility is an average decrease of almost $20 \%$ from its initial value. This decline in relative volatility allows the same conditions to be reached in a longer time, since the way to use the relative volatility to reduce the time required for the separation process is less straightforward, and this approach requires a greater amount of reflux to achieve the desired product concentration.

\section{CONCLUSIONS}

In this study, we proposed a simple procedure for the implementation of variable-reflux batch distillation based on the shortcut method developed by Sundaram and Evans (1993). The procedure is iterative and compares the desired value of the concentration of the light key component in the dome with the calculated value. The results obtained demonstrate that the proposal is appropriate and simpler than the methods developed by other authors (Diwekar, 1988; Zamar et al., 1998; Barolo and Guarise, 1999; Ehsani, 2002). Agreement between the results from this proposed procedure and the Diwekar shortcut model was excellent.

\section{ACKNOWLEDGEMENTS}

We thank the Universidad Autónoma del Carmen and Universidad Autónoma de Yucatán, Mexico, for the support given for the development of this study. Thanks are also due to PROMEP for providing financial resources through the PROMEP/103.5/10/5126 agreement.

\section{NOMENCLATURE}

$\begin{array}{llr}B & \text { bottom } & \mathrm{mol} \\ D & \text { product } & \mathrm{mol} \\ h k & \text { heavy key component } & \\ N & \text { number of stages } & \\ n c & \text { number of components } & \\ N_{\text {min }} & \text { minimum number of } & \\ & \text { separation stages } & \\ l k & \text { light key component } & \\ r & \text { reference component } & \mathrm{h} \\ R_{t} & \text { reflux ratio } & \mathrm{mol} / \mathrm{h} \\ R_{\min } & \text { minimum reflux ratio } \\ t & \text { time } & \\ V & \text { vapor flow } & \\ x & \text { liquid composition } & \end{array}$

\section{Index}

B Bottom 
hk heavy key component

lk light key component

\section{Greek Symbols}

$\alpha \quad$ relative volatility

$\phi \quad$ parameter of the Underwood

equations

\section{REFERENCES}

Barolo, M. and Guarise, G. B., Batch distillation of multicomponent systems with constant relative volatilities. Trans. IChemE, 74(A), 863 (1996).

Gmehling, J., Onken, U. and Arlt, W., Vapor-Liquid Equilibrium Data Collection. Dechema Chemistry Data Series, Dechema, Frankfurt (1987).

Diwekar, U. M., Simulation, design, optimization and optimal control of multicomponent batch distillation columns. Ph.D. Thesis, Indian Institute of Technology, Bombay, India (1988).

Diwekar, U. M. and Madhavan, K. P., Multicomponent batch distillation column design. Ind. Eng.
Chem. Res., 30(4), 713 (1991).

Diwekar, U. M. and Madhavan, K. P., Batch-dist: A comprehensive package for simulation, design, optimization and optimal control of multicomponent. Multifraction Batch Distillation Columns, Comput. Chem. Eng., 15(12), 833 (1991).

Ehsani, M. R., Prediction of the operating conditions in a batch distillation column using a shortcut method. Iran J. Chem. Chem. Eng., 21(2), 118 (2002).

Henley, E. J. and Seader, J. D., Separation Process Principles. Wiley, New York (1998).

Mujtaba, I. M., Batch Distillation: Design and Operation. Imperial College Press, London (2004).

Luyben, W. L., Some practical aspects of optimal batch distillation design. Ind. Eng. Chem. Process Des. Develop., 10(1), 54 (1971).

Shiras, R. N., Hanson, D. N. and Gibson, C. H., Calculation of minimum reflux in distillation columns. Ind. Eng. Chem., 2(5), 871 (1950).

Sundaram, S. and Evans, L. B., Shortcut procedure for simulating batch distillation operations. Ind. Eng. Chem. Res., 32(3), 511 (1993).

Zamar, S. D., Salomone, E. and Iribarren, O. A., Shortcut method for multiple task batch distillations. Ind. Eng. Chem. Res., 37(12), 4801 (1998). 